\title{
Deathzones and exponents: A different approach to incorporating mass loss in stellar evolution calculations
}

\author{
Lee Anne Willson ${ }^{1}$ \\ ${ }^{1}$ Department of Physics and Astronomy, Iowa State University, Ames, IA 50010, USA \\ email:lwillson@iastate.edu
}

\begin{abstract}
Observations tend to select mass loss rates near the critical rate, $\dot{M}_{\text {crit }}=M \dot{L} / L$. There are two reasons for this. In some situations, such as near the tip of the AGB, the mass loss rate is very sensitive to stellar parameters. In this case, stars with $\dot{M} \ll \dot{M}_{\text {crit }}$ have dustfree, hard-to-measure mass loss rates while stars with $\dot{M} \gg \dot{M}_{\text {crit }}$ do not survive very long and thus make up a small fraction of any sample. Selection effects dominate the fitting of empirical formulae; observations of mass loss rates tell us more about which stars are losing mass than about how a star loses mass. In other situations, such as for some of the stars along the RGB, a steady state situation occurs where the loss of mass leads to a decrease in mass loss rate while the evolutionary changes lead to an increase; the result is a steady state with $\dot{M}=\dot{M}_{c r i t}$. To determine the envelope mass and composition at the end of a phase of intensive mass loss requires stellar evolution models capable of responding on a time scale $\sim t_{K H}$ and thus, a new generation of stellar modeling codes.
\end{abstract}

Keywords. Stars: mass loss, stars: evolution

Stars lose mass, but only some of the time. We can distinguish mass loss, where the rate is high enough to affect the evolution of a star, from a stellar wind that carries only very small amounts of mass away. In most situations, mass loss occurs when $\dot{M} / M$ is not too much smaller than $\dot{L} / L$ or $\dot{R} / R$ or $T_{e f f} / T_{e f f}$, and over a limited range of parameters for a given star (Willson 2000, Willson 2006, Willson 2007).

Known epochs of mass loss for stars with masses from about 0.8 to about $8 M_{S u n}$ include the upper asymptotic giant branch (AGB) and, at least for some stars, the first ascent red giant branch (RGB). The stars near the tip of the AGB that are losing mass we identify as Mira variables; because the atmospheric structure is different for stars with strong winds, these stars have large visual magnitude variations as they pulsate. The stars with mass loss rates $>10^{-5} M_{S u n} / y r$ are also known as OHIR stars, IR bright stars with $\mathrm{OH}$ masers, as they have opaque circumstellar outflows.

Stellar evolution modeling has sought to include mass-loss by way of formulae found by fitting observations of mass loss rate expressed as functions of luminosity $L$, radius $R$, effective temperature $T_{\text {eff }}$, pulsation period $P$, or combinations of these. Other formulae have been proposed from theoretical studies of a particular mass loss mechanism. Mechanisms that have been modeled in particular detail include pulsation/dust mass loss for red giants (Bowen 1988, Bowen \& Willson 1991, Höfner 2007a, Höfner 2007b), and line driven winds for hot stars (Castor et al. 1975, Abbott 1982, Kudritzki et al. 1989).

Formulae derived in the usual manner by fitting mass loss rates vs. stellar parameters are not particularly useful for stellar evolution models, for two reasons: (a) they are dominated by severe selection effects; and (b) they are calibrated for a limited volume of parameter space where often at least one parameter (usually $M$ and/or $R$ ) cannot be 
well constrained by observations. For discussion of these points, see Willson (2000, 2006, 2007).

Here, I will discuss some general characteristics of mass loss episodes, then illustrate my main points with an analysis of mass loss on the AGB and the RGB.

\section{Why observations favor $M \approx M(\dot{X} / X)$}

Observations tend to yield $\dot{M} \approx M \dot{X} / X$ (where $X$ is $L$ or $R$ or $T_{\text {eff }}$ depending on the direction the star is evolving in the HR diagram) in two situations: (a) when the mass loss rate depends steeply on stellar parameters, often with positive feedback, or (b) when mass loss changes the situation so as to reduce the mass loss rate but evolutionary changes increase $\dot{M}$ - negative feedback or steady state mass loss.

In the first case, when mass loss rates depend steeply on stellar parameters, there is a narrow range of stellar parameters for which $\log \dot{M}=\log (M \dot{X} / X) \pm 1$. Below this range, mass loss rates have little effect on the evolution and may be hard to detect. Above this range, mass loss quickly removes the envelope of the star. Thus we will preferentially select and tabulate rates near $\dot{M}_{c r i t}=M \dot{X} / X$. This situation tends to apply where mass loss increases $R$ and mass loss rate increases with $R$ - such as near the tip of the AGB until the envelope mass is very small and the star leaves the AGB.

In this first case, it is useful to define the deathline as where $\dot{M}=M \dot{X} / X=\dot{M}_{\text {crit }}$ and the deathzone as where $\log \dot{M}=\log \dot{M}_{\text {crit }} \pm 1$. Nearly all the mass loss of evolutionary significance will take place in the deathzone when the mass loss rate depends steeply on stellar parameters.

In the second case, when the loss of a little mass changes the situation so as to reduce the mass loss rate, but evolutionary changes increase $\dot{M}$, then the mass loss rate will tend to the steady state value $\dot{M}=M \dot{X} / X\left(X=L\right.$ or $R$ or $\left.T_{e f f}\right)$ depending on the direction of evolution in the HR Diagram). In this case, the rate at which the star evolves due to internal processes determines the mass loss rate, and to predict the mass loss rate we need only know the rate of evolution, $\dot{X} / X$, for each mass $M$ from a stellar evolution code - we do not need to know the mechanism to predict the mass loss rate, as the steady $\dot{M} / M=\dot{X} / X$.

The mass loss terminates, in the first case, when the star has lost enough mass that the mass loss rate no longer increases with decreasing mass. For example, when an AGB star's envelope mass goes below a small value, the radius decreases with decreasing $M$. However, it will take the star a time on the order of the Kelvin-Helmholtz time $\left(t_{K H}\right)$ to adjust to a change in its mass, and thus the mass loss process will "overshoot" the simplest estimate by some amount. An order-of-magnitude estimate for the overshooting is $\dot{M}_{\text {max }} \times t_{K H}$; however, for such important questions as the evolution of post-AGB stars. the surface layers on white dwarfs, and the structure of pre-SNIIs, it will be necessary to use evolutionary models capable of responding on times shorter than $t_{K} H$ to derive $d \log R / d \log M$ for the process. Such codes are becoming available - for example, there is the Djehuty code being developed at LLNL (Bazán et al. 2003).

Finally, I note that a similar logic has long been applied to the evolution of binary systems with Roche lobe overflow, where the mass exchange is modeled using information about the evolutionary changes in each star and the effect of mass exchange, and sometimes also mass loss from the system, on the orbits of the two stars involved. (See Hjellming \& Webbnik 1987, Webbink 1976.) What I propose is we consider a similar approach to mass loss more generally, particularly since reliable observational determination of $\dot{M}(L, R, M, Z)$ is not available, and theoretical computations are not yet reliably predictive of such a formulae either. Both for complete understanding of the 
binary evolution and the single star evolution with mass loss, however, we need to be able to study the response of a star to changes of mass on time scales comparable to or less than the Kelvin-Helmholtz time. One significant difference is, though, that mass-loss on a dynamical time-scale, expected in some binary star systems, is not likely for the single star case. The difference is that in the binary case the orbit evolves on a dynamical time scale, so when mass loss leads to a decrease in the size of the orbit relative to the stellar radius this initiates very fast exchange. For the single star case we expect the changes to take place on time scales between the thermal or Kelvin-Helmhotlz timescale $t_{K H}$, and the nuclear time scale.

\section{Examples of the two cases}

\subsection{Mass loss at the tip of the $A G B$}

This case is one I have reviewed a number of times; see Willson (2000), Willson (2006), Willson (2007), Willson (2008). Because this is the main mass loss episode for most stars $\left(M=0.8\right.$ to $\left.8 M_{S u n}\right)$, there have been quite a few attempts to produce reliable mass loss laws from observations or from theory. Examples are reviewed in Willson (2007) and Willson (2008) taken from Vassiliadis \&Wood (1993), van Loon et al. (2005), Bryan, Volk \& Kwok (1990), Blöcker (1995), Schröder and Cuntz (2005), Baud \& Habing (1983), and Wachter et al. (2002). We have argued (Bowen \& Willson 1991, Willson 2000, Willson 2006, Willson 2007) that the episode is short-lived because the mass loss rate is very sensitive to stellar parameters. As the star evolves in $L$ and $R, \dot{M}$ increases from too small to notice to large enough to kill the star quickly in the course of just 2 to $4 x 10^{5}$ years. The mass loss prescription based on our 1995 grid of models $=\mathrm{BW}$ in what follows. Note that Blöcker (1995) used an earlier grid of models where the regulation of the driving amplitude in the models had not yet been set to an energy condition; for the 1995 grid and subsequent modeling we have implemented a maximum power condition that results in a steeper dependence on $L, R$, and $M$ (Willson 2000). A recent exploration with an independent code also provides support for this choice (LAWMa08).

If we reduce all the mass loss laws to a common basis, $\dot{M}(L, M, \alpha, Z)$, where $\alpha=\ell / H_{p}$, using evolutionary tracks with period-mass-radius relations and the definition of $T_{\text {eff }}$ as needed, then we can see (Figure 1 of Willson (2007), Figures $1 \& 2$ of Willson (2008)) that the relations all cross within a modest range of $\log \dot{M}$ and $\log L$, but that the slopes $d \log M d o t / d \log L$ (taken along the evolutionary track in this figure) are quite varied. This is what we expect if (a) the mass loss rate is highly sensitive to stellar parameters and (b) observations mainly pertain to stars within an order of magnitude or so of the critical rate, as noted above. Thus I have concluded that observations of mass loss rates tell us which stars are losing mass but (mostly) do not tell us much about how a given star will lose mass. The one possible exception to this rule is the Vassiliadis \&Wood (1993) $=\mathrm{VW}$ fit for Miras, $\log \dot{M}=-11.4+0.123 P$. Because they used a variable that is easily and accurately observed, pulsation period $P$, and because they restricted the fit to a homogeneous class of objects, Miras, they obtained a fit with a steep dependence of mass loss rate on stellar parameters near the deathline that is probably closer to having the right slope near the deathline than any of the other observational calibrations. Even in their case, however, not having observational determinations of the stellar masses leads to underestimating the sensitivity of the mass loss rate to the combined effects of increasing $R$ and decreasing $M$.

Theoretical models suffer also from selection effects. These include the choice of $L, R$ or $T_{\text {eff }}$ for which to run models; the choice of modeling parameters (such as mixing length 
or $R$ for a given $L, M$, for a giant); "piston" amplitude for most pulsating atmosphere modeling; dust condensation formulation; handling of cooling and heating by interaction with the radiation field; and treatment of the radiative transfer problem for a dynamical atmosphere). Some choices will produce more shallow slopes and others, a steeper slope. For example, the piston amplitude may be taken to have the same velocity amplitude $\triangle v$, the same $\triangle v^{2}$, or the same peak power (taking into account work done on the atmosphere by the piston at some phases and by the atmosphere on the piston at other phases).

Typically, the power going into driving the wind, roughly $\frac{1}{2} \dot{M}\left(v_{e s c}^{2}+v_{\infty}^{2}\right)$, is many orders of magnitude below the stellar luminosity, and so, it is a small residual of much more energetic processes. Without violating energy conservation the energy associated with mass loss can easily vary around a typical $10^{-5} L_{*}$ (corresponding to the Reimers' relation) by several orders of magnitude. The choice of which models to run and how to model the processes giving rise to mass loss can influence the derived formula, just as the selection of which stars to study can affect the empirical relations.

The mass loss process along the AGB is an example of positive feedback - as $M$ decreases, $R$ increases, and both changes increase the mass loss rate. The duration of the mass loss phase is thus determined by how steeply the mass loss rate depends on $M, L, R$ and/or $T_{\text {eff }}$. Reducing the variables for a given $M$ to a single one, $L$, taken along the evolutionary track, and finding the slope at the deathline, gives values that may be compared directly with each other at a given $L$ near the deathline. The duration of the mass loss phase is $<(L / \dot{L}) /(d \log \dot{M} / d \log L) \approx 10^{6}$ years $/(d \log \dot{M} / d \log L)$. The duration is $>10^{6}$ years for most of the formulae we have examined, but $\sim 4 x 10^{5}$ years for the VW and BW mass loss formulae; observations support a value closer to 2 to $4 x 10^{5}$ years (Jura and Kleinmann 1992).

When the envelope mass reaches a critical value that depends on the core mass, further reduction in the envelope mass will decrease the radius of the star (e.g. Frankowski 2003, Iben \& Renzini 1983). Now, both evolution and mass loss have the same effect - reduction of the envelope mass. Evolution also increases $L$, but relatively slowly, so the mass loss rate should quickly go to zero as the star shrinks at nearly constant $L$. Again, we expect the shrinkage to take at least $t_{K H}$, so we expect the mass loss to end with an envelope mass smaller than the critical value obtained from quasi-equilibrium stellar models. Note that $t_{K H}$ for these extreme giants is only a few decades, while the dynamical time is around one year; Ostlie \& Cox (1986) (Figure 4) showed that the growth rates of pulsation modes were quite sensitive to the ratio $t_{d y n} / t_{K H}$ and relatively large for small ratios, so some erratic pulsation may be expected at/near the end of this mass loss episode.

\subsection{Mass loss along the $R G B$}

From the appearance of cluster HR diagrams with well-populated horizontal branches it is apparent that cluster stars of a given initial mass do not all lose the same amount of mass along the RGB. Recent observations by Origlia et al.(2007) have confirmed that the mass loss rates of red giants in 47 Tuc are not all the same at a given $L$, with 10 to $30 \%$ of red giants at a given $L$ showing an IR excess. These also show that mass loss occurs within a single cluster at a range of values of $L$. The rates satisfy $\dot{M} \approx \dot{M}_{\text {crit }}$ also for the RGB stars. Because this mass loss occurs over a range of $L$ for a given initial $M$, this is most likely to be a case of a steady state mass-loss process, i.e. one where the loss of a little mass leads to changes that reduce the mass loss rate. Since, on the RGB, decreasing $M$ increases $R$, and since mass loss rates generally increase with $R$, this is unlikely to be the result of a mechanism operating for a single star. We are investigating a mechanism for mass loss that involves a low mass companion with a star with relatively rapidly increasing radius (subgiant or giant); in this case, the companion can't enforce 
synchronous rotation, and therefore the standard Roche lobe analysis does not apply. For details, see Wang et al. (this volume) and Struck et al. (in prep).

\section{Recipe for handling mass loss in stellar evolution calculations}

Above I have argued that (a) we do not have a reliable mass loss law for important episodes of mass loss in the life of many stars; and (b) typically, interesting mass loss happens near $\dot{M}=\dot{M}_{\text {crit }}$ where $\dot{M}_{\text {crit }}$ may be determined directly from evolutionary models. This suggests a very different approach for incorporating mass loss into stellar evolution models:

(a) Include mass loss only where observations say it occurs - do not extrapolate from limited ranges of stellar parameter space.

(b) Where mass loss matters, use duration, amplitude, and distribution data to estimate the exponents (slopes $d \log \dot{M} / d \log X$ where $X=L, R, T_{\text {eff }}$ or a combination taken along the dominant direction of evolution.

(c) Calculate, from models, $M \dot{L} / L=\dot{M}_{c r i t}$. See how the observed mass loss rates are distributed with respect to this critical rate.

[i] If the distribution is broad, and the stars are located near or at an extreme value of stellar parameters, suspect a "deathline/deathzone" situation and proceed accordingly.

[ii] If the distribution is narrow, and the stars distributed over a range of stellar parameters, suspect a steady-state mass loss process, and evaluate possible mechanisms accordingly.

(d) Compute a grid of models without massloss that cover all the parameter space occupied by stars in the appropriate population.

(e) Where mass loss is likely, use an appropriate code to determine the (local) exponents $d \log R / d \log M, d \log L / d \log M$, and $d \log T_{e f f} / d \log M$. Based on the argument, above, that the mass loss time scale will be close to the nuclear time scale at steady state or at the deathline, and normally $t_{d y n} \ll t_{K H} \ll t_{n u c l e a r}$, an ordinary evolutionary code may be used to find these exponents for mass loss rates up to the critical value, while a code capable of modeling processes faster than the nuclear scale will be needed for the high-mass-loss end of the deathzone.

$(f)$ Supplement the no-mass-loss grid with the results of the mass loss analysis. This may truncate the evolution, or shift subsequent evolution to a new track, or introduce a dispersion in the results to be expected from an initially homogeneous population (with heterogeneous rotation, duplicity or planet families).

\section{Conclusions and the future}

To a great extent we can separate two hard problems: The incorporation of mass loss into stellar evolution, and the determination of mass loss rates and mass loss mechanisms for various classes of star. This parallels the logic that has been used to model the evolution of binary star systems, but instead of a condition of Roche lobe overflow to determine when mass loss occurs, we will use observations of which stars are losing mass at interesting rates, and constraints from the observed duration, amplitude, and distribution of mass loss rates in each episode. This approach will suffice to derive initial-final mass relations, and thus also to compute the colors or composite spectra of populations of stars. It will not suffice to tell us the precise envelope mass at which the mass loss process stops, however, nor do traditional stellar evolution models suffice. For such nuances we will need 
to study the response of a star to mass loss with codes capable of following changes on time scales shorter than the Kelvin-Helmholtz time.

\section{References}

Abbott, D. C. 1982, ApJ, 259, 282

Baud, B. \& Habing, H. J. 1983 aap, 127, 78-83

Bazán, G., et al. 2003, 3D Stellar Evolution, 293, 1

Blöcker, T. 1995, aap, 297, 727

Bowen, G. H. \& Willson, L. A. 1991, ApJ, 375, L53

Bowen, G. H. 1988, ApJ, 329, 299

Bryan, G., Volk, K., \& Kwok, S. 1990, ApJ, 365, 301-211

Castor, J. I., Abbott, D. C., \& Klein, R. I. 1975, ApJ, 195, 157

Frankowski, A. 2003, aap, 406, 265

Höfner, S., Gautschy-Loidl, R., Aringer, B., \& Jørgensen, U. G. 2003, aap, 399, 589

Höfner, S. \& Anderson, A. 2007, A\&SA, 465, L39-L42

Höfner, S. 2007, Why Galaxies Care About AGB Stars: Their Importance as Actors and Probes, 378,145

Hjellming, M. S., \& Webbnik, R. F. 1987, ApJ, 318, 794

Iben, I., Jr. 1984, ApJ, 277, 333

Iben, I., Jr. \& Renzini, A. 1983, ARAA, 21, 271-342

Jura, M. \& Kleinmann, S. G. 1992, ApJS, 79, 105-121

Kudritzki, R. P., Pauldrach, A., Puls, J., \& Abbott, D. C. 1989, aap, 219, 205

Mattson, L., Wahlin, R., H'ofner, S., \& Eriksson, K. in press, A\&SA, ArXiv 0804.2482

Origlia, L., Rood, R. T., Fabbri, S., Ferraro, F. R., Fusi Pecci, F., \& Rich, R. M. 2007, ApJL, $667, \mathrm{~L} 85$

Ostlie, D. A. \& Cox, A. N. 1986, ApJ, 311, 864

Reimers, D. 1975, Mem. Soc. Roy. Sci. Liège, 8, 369

Schröder, K.-P. \& Cuntz, M. 2005, ApJ, 630, L73

van Loon, J. T., Cioni, M.-R. L., Zijlstra, A. A., \& Loup, C. 2005, aap, 438, 273

Vassiliadis, E. \& Wood, P. R. 1993, ApJ, 413, 641

Volk, K. and Kwok, S. 1988, ApJ, 331, 435-462

Wachter, A., Schröder, K.-P., Winters, J. M., Arndt, T. U., \& Sedlmayr, E. 2002, aap, 384, 452

Webbink, R. F. 1976, ApJ, 209, 829

Willson, L. A. 2000, ARAA, 38, 573

Willson, L. A. 2006, ESO Astrophysics Symposia, Springer, Planetary Nebulae Beyond the Milky Way, 99

Willson, L. A. 2007, ASP Conference Series, Vol. 378, Why Galaxies Care About AGB Stars: Their Importance as Actors and Probes, p211

Willson, L. A. 2008, ASP Conference Series, The BIggest Baddest Coolest Stars, in press.

Willson, L. A. \& Kim, A. 2004, ASP Conf. Ser. 313: Asymmetrical Planetary Nebulae III: Winds, Structure and the Thunderbird, 313, 394

\section{Discussion}

PodsiAdLOWsKI: I completely agree with you that AGB stars must have a deathline, and there is a simple theoretical reason for it, as pointed out many years ago by Han, Podsiadlowski and Eggleton (1994), going back to a suggestion by Ziolkowski and Paczynski, and that is that at this point the AGB star envelopes start to have positive binding energies. This explains both the initial to final mass relation and the whitedwarf mass distribution. Indeed, I think that this must be the ultimate cause for the Mira variability. 
WILlson: Several things happen near the AGB tip - the question is which happens first. The initial-final mass relation is not the best constraint - final $\mathrm{L}$ is better since $\mathrm{L}-\mathrm{M}_{c}$ relations can be shown to be wrong.

WoITKE: What can we learn from the structure of planetary nebulae, where the final mass loss history is imprinted? Due to thermal pulses, the stars may cross the "death zone" several times during the final evolution, producing multiple shells (e.g., Wachter et al.; Schröder et al.).

WiLlson: (1) I think there is useful information in these observations, but we are not yet ready to extract the meaning. (2) We need more sophisticated models to extract the information of $\dot{M}$-history from PN. There are complications due to binaries. 